Correspondence: D McLeod,

Tel: + 44161276 5645;

Fax: + 441612736354

E-mail: david.mcleod@man.ac.uk

Eye (2005) 19, 1350-1351. doi:10.1038/sj.eye.6701795; published online 28 January 2005

Sir,

\section{Reply to McLeod}

We would like to thank to Dr. McLeod for his constructive contributions to our paper describing retinal vascular occlusions in two cases with homocystinaemia. ${ }^{1}$ In our paper, we have primarily focused on the finding of homocystinaemia as a cause of vascular occlusions in young patients; however, we have not discussed the probable mechanisms of association of arterial and venous obstructive disease. Three different clinical syndromes have been suggested describing simultaneous arterial and venous obstructions of the retina including; the combination of central retinal artery and vein obstruction; combined occlusion of central retinal vein and cilioretinal artery; and combined branch retinal artery and central retinal vein obstruction. ${ }^{2}$ We agree with Dr McLeod in that the arterial obstruction may actually be a relative hypoperfusion of the cilioretinal arteries secondary to increased retinal venous pressure. ${ }^{3,4}$ Although this dependent occlusion is the most probable explanation, therapeutic lowering of the intraocular pressure was attempted in the presented case considering the other possibilities like two simultaneous (but separate) occlusions which may be the case in a patient with homocystinaemia.

\section{References}

1 Ozdek S, Yulek F, Gurelik G, Aydin B, Hasanreisoglu B. Simultaneous central retinal vein and retinal artery branch occlusions in two patients with homocystinaemia. Eye 2004; 18: 942-945.

2 Singh AJ. Branch retinal artery obstruction with simultaneous central retinal vein occlusion. Eye 2001; 15: 225-227.

3 McLeod D, Ring CP. Cilio-retinal infarction after retinal vein occlusion. Br J Ophthalmol 1976; 60: 419-427.

4 McLeod D. Central retinal vein obstruction with cilio-retinal infarction. Eye 2003; 17: 283.

S Ozdek

Ophthalmology Department, School of Medicine, Gazi University, Ankara, Turkey

Correspondence: S Ozdek, Isci Blokları mah. 31. Cad., Ozgur Anıl sit. A-Blok No: 30, 100.YIL
06530, Ankara, Turkey

Tel/Fax: +90312 2125794

E-mail: sozdek@gazi.edu.tr

Eye (2005) 19, 1351. doi:10.1038/sj.eye.6701796; published online 21 January 2005

Sir,

Diode laser trans-scleral cyclophotocoagulation in the management of glaucoma in patients with long-term intravitreal silicone oil

We read with great interest the article by Sivagnanavel et al. ${ }^{1}$ We will be most grateful if the authors can help to clarify a few points.

In this series, the authors described a significant number of subjects losing vision after trans-scleral cyclophotocoagulation (TSCPC). They felt that the loss was not quantifiable because of the poor visual acuity. They concluded that TSCPC failed to protect against visual loss in the long term. We may like to be aware that it is the natural course of their disease to lose vision. Hence, they may like to compare with the rate of visual loss in the TSCPC-treated group with a control group having similar glaucoma associated with long-term silicone oil placement, and who were treated with other modalities. It may be of interest for the authors to attempt quantification with Logmar (Logarithm of Minimum Angle of Resolution) chart, or express the percentage of eyes losing 2 or more lines.

If the eight cases of ocular comorbidity were excluded, the success rate can actually be raised to $50 \%$. As the authors rightly pointed out, the prolonged placement of silicone oil before treatment (mean duration of oil before TSCPC was 33.7 months (range 1-113 months, $\mathrm{SD}=26.9)$ ), and prolonged duration before successfully bringing down the IOP ( $53 \%$ of the patients took 450 days to reduce the IOP to below $21 \mathrm{mmHg}$ ), might be factors contributing to the low overall success rate. We may like to be aware of the fact that the success and failure definitions were slightly different among different studies so that the rates may not be accurately comparable with each other. ${ }^{2}$

The authors did possess evidence in support of the efficacy of the TSCPC: the average number of IOP-lowering medications prior to TSCPC was 2.6 (range 1-5). This was reduced to 1.0 (range 0-3) following TSCPC at final follow-up. Moreover, the authors regarded the procedure to be very safe with few side effects. At the time of writing, as far as we know, there is an absence of strong data in the literature supporting other modalities of treatment in the same 